\title{
A NEW RESULT CONCERNING THE STRUCTURE OF ODD PERFECT NUMBERS
}

\author{
PETER HAGIS, JR. AND WAYNE L. McDANIEL
}

Abstract. It is proved here that an odd number of the form $p^{\alpha} s^{6}$, where $s$ is square-free, $p$ is a prime which does not divide $s$, and $p$ and $\alpha$ are both congruent to 1 modulo 4 , cannot be perfect.

A positive integer $n$ is said to be perfect if

$$
\sigma(n)=2 n,
$$

where $\sigma(n)$ denotes the sum of the positive divisors of $n$. To date 24 perfect numbers have been discovered, all of which are even. Although no one knows whether or not any exist, many interesting results have been obtained concerning the structure of odd perfect numbers. The oldest of these goes back to Euler who showed that if $n$ is an odd perfect number then

$$
n=p^{\alpha} p_{1}^{2 \beta_{1}} p_{2}^{2 \beta_{2}} \cdots p_{t}^{2 \beta_{t}}
$$

where $p, p_{1}, \cdots, p_{t}$ are distinct odd primes and $p \equiv \alpha \equiv 1(\bmod 4)$. In 1937 Steuerwald [5] proved that not all of the $\beta_{i}$ in (2) can equal 1. Four years later Kanold [1] showed that the $\beta_{i}$ cannot all be equal to 2 . In the same paper he also proved that the numbers $2 \beta_{i}+1(i=1,2, \cdots, t)$ cannot have as a common divisor any of the numbers $9,15,21$ or 33 . Recently McDaniel [3] has generalized these results by proving that 3 cannot be a common divisor of the $2 \beta_{i}+1$. The purpose of the present paper is to show that the $\beta_{i}$ in (2) cannot all be equal to 3 . Thus, we shall prove the following result.

THEOREM. If $n=p^{\alpha} p_{1}^{6} p_{2}^{6} \cdots p_{t}^{6}$ is an odd number such that $p \equiv \alpha \equiv 1$ $(\bmod 4)$, then $n$ is not perfect.

Our method of proof requires us to find the prime factors of some very large numbers. This part of the research was done using the CDC 6400 at the Temple University Computing Center.

Received by the editors May 26, 1971.

AMS 1970 subject classifications. Primary 10A20.

Key words and phrases. Odd perfect numbers, prime decomposition, exponents.

(c) American Mathematical Society 1972 
We begin by stating a result concerning cyclotomic polynomials. The proof may be found in [4].

Proposition 1. $x^{m}-1=\prod_{d \mid m} F_{d}(x)$ where $F_{d}(x)$ is the dth cyclotomic polynomial.

We shall also require the following facts concerning odd perfect numbers. The first appears in [2]. The second is due to Kanold [1].

Proposition 2. If $n$ is an odd perfect number, then $105 \nmid n$.

Proposition 3. If $n$ is an odd perfect number as in (2) and $s$ is a common divisor of the numbers $2 \beta_{i}+1(i=1,2, \cdots, t)$, then $s^{4} \mid n$.

We now assume that the $n$ of our theorem is perfect and shall reach a contradiction. Using the well-known formula for the $\sigma$-function, we see from (1) and Proposition 1 that $2 n=\sigma\left(p^{\alpha}\right) \prod_{i=1}^{t} F_{7}\left(p_{i}\right)$. Therefore, every prime divisor of $F_{7}\left(p_{i}\right)$ is a divisor of $n$. Also, since $\alpha$ is odd, it follows that $(p+1) \mid \sigma\left(p^{\alpha}\right)$, so that every odd prime dividing $p+1$ also divides $n$. From Proposition 3 we deduce that $7^{4} \mid n$ and therefore that $F_{7}(7)=29.4733$ divides $n$ (note that 4733 is prime). If $p=29$, then $3 \cdot 5 \cdot 7 \mid n$ which contradicts Proposition 2. Therefore, $p \neq 29$ and $F_{7}(29)=7 \cdot 88009573=7 Q$ divides $n$ where $Q$ is a prime. Since $Q \equiv 1(\bmod 4), Q$ may or may not be equal to $p$. We consider these possibilities separately.

If $Q \neq p$, then $F_{7}(Q) \mid n$. A search for "small" prime factors of $F_{7}(Q)$ yielded $7,29,43,71$. Since neither 43 nor 71 is congruent to 1 modulo 4 , neither is equal to $p$. The prime factorization of $F_{7}(43)$ is $7 \cdot 5839 \cdot 158341$ while $883 \mid F_{7}(71)$. We are now certain that the following eight primes divide $n: 29,43,71,883,4733,5839,158341,88009573$. All are congruent to 1 modulo 7 and at most one (either 4733 or 158341) can be $p$. Since $x \equiv 1$ $(\bmod 7)$ implies $F_{7}(x)=1+x+x^{2}+\cdots+x^{6} \equiv 0(\bmod 7)$ we conclude that $7^{7} \mid n$. But this contradicts the fact that $7^{6} \| n$.

If $Q=p$ then $n$ is divisible by $(p+1) / 2=53 \cdot 830279=53 R$ where $R$ is a prime. The prime factors of $F_{7}(R)$ which do not exceed 10000 are 43 and 1709 , while the prime factorization of $F_{7}(53)$ is $29 \cdot 778986167$. We now know that the seven primes $29,43,1709,4733,5839,158341,778986167$, each different from $p$ and each congruent to 1 modulo 7, divide $n$. As before, $7^{7} \mid n$ which is a contradiction. This completes the proof of our theorem.

An examination of the details of our argument shows that we have also established the following result.

COROLLARY. If $n=p^{\alpha} 7^{6} p_{2}^{2 \beta_{2}} \cdots p_{t}^{2 \beta_{t}}$ is an odd number such that $7 \mid\left(2 \beta_{i}+1\right)$ for $i=2, \cdots, t$ and $p \equiv \alpha \equiv 1(\bmod 4)$, then $n$ is not perfect. 


\section{REFERENCES}

1. H.-J. Kanold, Untersuchungen über ungerade vollkommene Zahlen, J. Reine Angew. Math. 183 (1941), 98-109. MR 3, 268.

2. U. Kühnel, Verschärfung der notwendigen Bedingungen für die Existenz von ungeraden vollkommenen Zahlen, Math. Z. 52 (1949), 202-211. MR 11, 714.

3. W. L. McDaniel, The non-existence of odd perfect numbers of a certain form, Arch. Math. 21 (1970), 52-53. MR 41 \#3369.

4. I. Niven, Irrational numbers, Carus Math. Monographs, no. 11, Math. Assoc. of America, distributed by Wiley, New York, 1956. MR 18, 195.

5. R. Steuerwald, Verschärfung einen notwendigen Bedingung für die Existenz einen ungeraden vollkommenen Zahl., S-B. Math.-Nat. Abt. Bayer. Akad. Wiss. 1937, 68-72.

Department of Mathematics, Temple University, Philadelphia, Pennsylvania 19122

Department of Mathematics, University of Missouri-St. Louis, St. Louis, MissoURI 63121 